\title{
Fuentes de información digitales en los centros de documentación de prensa: las bases de datos comerciales
}

\author{
Por Javier Guallar Delgado y Anna Cornet Casals
}

\begin{abstract}
Resumen: Se describe en primer lugar el contexto general de la utilización de las fuentes de información externas en soporte digital en los centros de documentación de los medios de comunicación de prensa escrita. A continuación se presentan y comentan los resultados de una encuesta realizada entre los principales medios españoles sobre los recursos electrónicos externos comerciales de que disponen. Por último, se analiza brevemente cada producto informativo.
\end{abstract}

Palabras clave: Documentación periodística, Fuentes de información, Centros de documentación, Bases de datos, Recursos electrónicos, Recursos digitales, Prensa, Diarios.

\section{Title: Digital information resources in press docu- mentation centres: commercial databases}

Abstract: The article begins by providing an overview of the use of external digital information resources in press documentation centres. Secondly, the results of a survey concerning the external commercial digital resources available to the principal Spanish media are presented and discussed. Finally, each information resource is briefly analysed.

Keywords: Press documentation, Information resources, Documentation centres, Databases, Electronic resources, Digital resources, Press, Newspapers.

\section{Propósito y estructura del artículo}

En la bibliografía de ámbito español sobre fuentes de información para el sector de los medios de comunicación existen diversos estudios de interés que incluyen repertorios más o menos extensos de recursos informativos; por ejemplo, Cebrián (1997, 2002), Pons (1995), Chacón-García Jiménez (2001), Giménez Toledo (2002) o Pareja (2002). Sin embargo, son más escasos los estudios empíricos que ofrezcan datos sobre la presencia de tales recursos en los centros de documentación de prensa. Entre éstos últimos, se pueden destacar los trabajos de Fuentes (1991, 1994) y Fuentes-Conesa (1994), que describen las prácticas documentales en diversos servicios de documentación de medios de comunicación españoles, si bien los datos más recientes aportados se remontan diez años. Existen, eso sí, algunos estudios de caso sobre centros concretos, pero no trabajos que aporten una mirada de conjunto sobre el sector con datos actuales.

Por otra parte, en lo que a este tema se refiere, los propios profesionales de los servicios de documentación de los medios -es nuestra impresión personal- no suelen tener una visión de conjunto del sector más allá de los límites impuestos por los hábitos y los medios disponibles en su centro de trabajo: si bien tienen un profundo conocimiento de aquellos productos con los que trabajan, no conocen con detalle la forma de trabajo de los demás centros.

El origen de este artículo se encuentra en estas dos consideraciones previas. Por ello, el propósito del mismo es el de aportar algunos datos sobre la presencia "de facto" de fuentes de información externas en las unidades documentales de los diarios españoles. Con este objetivo se ha centrado el ámbito de estudio en una tipología concreta de fuentes digitales externas, las bases de datos comerciales, y se ha optado por analizar una muestra seleccionada de centros documentales mediante una encuesta. Los resultados que se muestran tienen 
por tanto un alcance concreto $\mathrm{y}$ perfectamente delimitado.

\section{«Los periodistas re- quieren a los centros de documentación in- formaciones total- mente heterogéneas ya que cualquier te- ma es susceptible de ser noticia»}

El trabajo se presenta estructurado en los cuatro apartados siguientes. El primero de ellos, "Fuentes de información en los centros de documentación de pren$\mathrm{sa}^{\text {" }}$ pretende situar el contexto de la utilización de los productos objeto del estudio. El apartado 3, "utilización de bases de datos comerciales en los principales diarios españoles", constituye el cuerpo central del estudio. En él se dan a conocer los resultados de la encuesta realizada y se comentan atendiendo al nivel de suscripciones de los centros de documentación, al grado de presencia de las bases de datos y a la antigüedad de contratación de las mismas. Se comparan asimismo los datos obtenidos ahora con los conocidos de hace 10 años. Una valoración sintética de todo ello se muestra en el apartado 4 de conclusiones. Por último, el anexo "Descripción de las bases de datos" es un repertorio comentado de estos recursos para centros de documentación de prensa. Nótese que la peculiariedad del mismo con relación a otros existentes es que el listado de fuentes no obedece a una selección de los autores sino a la práctica diaria de los centros consultados.

\section{Fuentes de información en los centros de documentación de prensa}

Los centros de documentación de los medios de comunicación, y en concreto de los medios de prensa escrita objeto de este estudio, ofrecen a sus usuarios (mayoritariamente, los redactores de los propios medios), las informaciones que éstos les requieren para la elaboración diaria de un producto informativo de calidad. Éstas tienen una doble procedencia: interna y externa.

Los redactores de prensa necesitan documentarse constantemente con todo tipo de informaciones para el desarrollo de su trabajo. Por una parte, precisan acceder a las elaboradas previamente por ellos mismos o por sus compañeros de redacción en un proceso continuo de retroalimentación que forma parte de la esencia de la información periodística. En segundo lugar, y no por ello menos importante, requieren a los centros de documentación informaciones de carácter totalmente heterogéneo que abarcan desde aquellas que son propiamente periodísticas, como pueden ser noticias publicadas en otros medios, a las específicas de temas económicos, legislativos, históricos, científicos o de cualquier materia imaginable, ya que cualquier tema es susceptible de ser noticia.

Para atender con eficacia este segundo grupo de necesidades informativas, los centros de documentación de prensa generalmente cuentan con una variedad de fuentes de información, gratuitas y de pago, cuyo nivel de presencia suele estar relacionado con la capacidad e importancia de la empresa periodística.

Los centros han contado tradicionalmente con una colección de obras de referencia y especializadas como un recurso básico y han acudido asimismo a toda una serie de fuentes informales (contactos telefónicos o de otro tipo con expertos, con otros centros de documentación, etc.). Sea cual fuere la fuente utilizada, el objetivo es siempre dar respuesta a una necesidad de información de la manera más precisa y rápida posible.

En la última década aproximadamente, en los centros de los diarios españoles - con cierto retraso respecto a otros países, particularmente los anglosajones-, se ha añadido toda una serie de fuentes de información distribuidas en soporte digital a las tradicionales de las obras de consulta (papel y microformas) y a las fuentes informales.

Dado que en la terminología seguida para designar los nuevos soportes informativos se utilizan habitualmente los términos digital y electrónico, podemos denominar a este grupo de productos indistintamente como fuentes de información digitales, fuentes de información electrónicas, recursos digitales, recursos electrónicos o recursos-e, etc. Con cualquiera de estos apelativos nos estamos refiriendo a productos informativos, elaborados específicamente o no para el sector de los medios de comunicación, que tienen una distribución digital.

Hace una década los recursos digitales que los centros de documentación de prensa podían adquirir eran bases de datos comerciales con tecnología propia, a las cuales se accedía en línea, como era el caso de Efedata de la agencia de prensa $E F E$ o de las diferentes bases de datos de la agencia Reuters En aquellos momentos la conexión en línea se establecía directamente con el servidor de cada empresa. Posteriormente se añadió a esta forma de distribución la comercialización de productos informativos sobre soportes ópticos (cd-rom), como hicieron el Boletín Oficial del Estado (BOE) o los diarios $A B C$, La vanguardia y El periódico.

Tras la eclosión de la World Wide Web a partir de la mitad de la década de los años noventa del si- 
glo pasado, las bases de datos comerciales que tenían sistemas propios iniciaron una migración hacia los estándares web que se iban imponiendo hasta producirse la práctica desaparición de los programas propios. La web es la forma de distribución seguida mayoritariamente por los productos digitales de pago en la actualidad, también por encima de la comercialización en soporte óptico.

Paralelamente, el impacto masivo de internet ha conllevado la proliferación de fuentes de información gratuitas, que son habitualmente conocidas en el ámbito de la documentación como recursos web, recursos de internet, recursos digitales o recursos electrónicos, añadiéndoles o no el calificativo de gratuitos.

Resumiendo, en la actualidad los recursos de información digitales que utilizan los centros de documentación de prensa son de dos tipos:

-De pago, conocidos generalmente como bases de datos comerciales, distribuidos en su mayoría por internet y en menor grado en cd-rom, que son adquiridos por el centro de documentación por sus- cripción anual renovable o por cualquier otro sistema de pago; y

- Gratuitos que, debido a su incesante aumento, requieren de un importante esfuerzo de conocimiento y selección por parte del centro.

«Dos productos tienen una implantación muy alta en los centros de documentación de prensa: Efedata está presente en 8 de los 10 diarios consultados y $M y$ news on line en 7»

En este estudio se indaga cuál es la presencia actual del primer grupo de recursos-e en los departamentos de documentación de los diarios españoles y se deja para un próximo trabajo el estudio del segundo grupo de fuentes gratuitas.

\section{Utilización de bases de datos comerciales en los principales diarios españoles}

Para conocer cuáles son las fuentes de información digitales de pago que se utilizan en estos momentos en las unidades de docu- mentación de prensa escrita en España, se ha procedido a realizar una encuesta a varios de estos centros. Dado que un estudio exhaustivo en todos los centros de los diarios españoles desbordaría los límites de este trabajo, se ha elegido una muestra significativa de los mismos. Se han estudiado los diarios que tienen los índices de audiencia más altos de España en la presunción de que las empresas informativas más potentes pueden presentar un nivel superior de utilización de productos de pago al de otras empresas informativas de carácter más modesto.

Se cursó una encuesta a los departamentos de documentación de los diez diarios españoles de información general de más audiencia, tomando como referencia los datos del último Estudio general de medios (EGM) disponible, de mayo de 2003: ABC, Diario El correo, El mundo, El país, El periódico, Heraldo de Aragón, La nueva Espa$\tilde{n} a, L a$ razón, $L a$ vanguardia y $L a$ voz de Galicia.

A los centros de documentación de estos medios se les ha preguntado por las bases de datos digitales de pago de que disponen y por la fecha de su adquisición. Los

\begin{tabular}{|c|c|c|c|c|c|c|c|c|c|c|c|}
\hline \multirow[t]{2}{*}{ Bases de datos } & \multicolumn{11}{|c|}{ Centros de documentación } \\
\hline & $\begin{array}{c}A B \\
C\end{array}$ & $\begin{array}{c}\text { Diario } \\
\text { El } \\
\text { Correo }\end{array}$ & $\begin{array}{c}\text { El } \\
\text { Mundo }\end{array}$ & $\begin{array}{c}\text { El } \\
\text { País }\end{array}$ & $\begin{array}{c}\text { El } \\
\text { Periódico }\end{array}$ & $\begin{array}{c}\text { Heraldo } \\
\text { de } \\
\text { Aragon }\end{array}$ & $\begin{array}{c}\text { La } \\
\text { Razón }\end{array}$ & $\begin{array}{c}\text { La } \\
\text { Nueva } \\
\text { España }\end{array}$ & $\begin{array}{c}\text { La } \\
\text { Vanguardia }\end{array}$ & $\begin{array}{c}\text { La Voz } \\
\text { de } \\
\text { Galicia }\end{array}$ & total \\
\hline$A B C$ & & & $\mathrm{x}$ & & & & $\mathrm{x}$ & & & & 2 \\
\hline Axesor (antes Infotel ) & & $\mathrm{x}$ & $\mathrm{x}$ & & $\mathrm{x}$ & & & & & & 3 \\
\hline Bloomberg & & & & & & & & & $x$ & & 1 \\
\hline$B O E$ & $\mathrm{x}$ & & & $\mathrm{x}$ & $\mathrm{x}$ & & & & $\mathrm{x}$ & & 4 \\
\hline Dogc & & & & & $\mathrm{x}$ & & & & $\mathrm{x}$ & & 2 \\
\hline Efedata & $\mathrm{x}$ & $\mathrm{x}$ & $\mathrm{x}$ & $\mathrm{x}$ & $\mathrm{x}$ & & $\mathrm{x}$ & & $x$ & $\mathrm{x}$ & 8 \\
\hline El País & $\mathrm{x}$ & & $\mathrm{x}$ & & & & $\mathrm{x}$ & & & & 3 \\
\hline Factiva (antes Reuters ) & & & $\mathrm{x}$ & $\mathrm{x}$ & $\mathrm{x}$ & & & & $\mathrm{x}$ & & 4 \\
\hline Fichero Altos Cargos & $x$ & & & & & & & & & & 1 \\
\hline Informa & & & $\mathrm{x}$ & & & & & & & & 1 \\
\hline La Vanguardia & & & $\mathrm{x}$ & & & & & & & & 1 \\
\hline LexisNexis & & & $\mathrm{x}$ & & & & & & & & 1 \\
\hline Mynews on line & $\mathrm{x}$ & $\mathrm{x}$ & $\mathrm{x}$ & $\mathrm{x}$ & $\mathrm{x}$ & & $\mathrm{x}$ & & $x$ & & 7 \\
\hline $\begin{array}{l}\text { Registro Mercantil } \\
\text { Central }\end{array}$ & & & $x$ & & $x$ & & & & & & 2 \\
\hline The Wall Street journal & & & $\mathrm{x}$ & & & & & & & & 1 \\
\hline $\begin{array}{l}\text { Westlaw (antes } \\
\text { Aranzadi ) }\end{array}$ & $x$ & & $x$ & & & & & & & $x$ & 3 \\
\hline total & 6 & 3 & 12 & 4 & 7 & 0 & 4 & 0 & 6 & 2 & 44 \\
\hline
\end{tabular}

Tabla 1. Las bases de datos comerciales en los centros de documentación de los principales diarios españoles 
datos obtenidos en la encuesta corresponden a los meses de julio y agosto de 2003 y se presentan en las tablas 1 y 2 .

La tabla 1 muestra la relación de bases de datos comerciales contratadas por cada uno de los centros mencionados y la tabla 2 el grado de antigüedad de su adquisición.

Es preciso matizar que en los datos presentados no se han considerado algunas obras de referencia en formato digital como diccionarios, enciclopedias o anuarios. Ante la diferencia de criterios en las respuestas de los centros a la hora de ser consideradas como bases de datos comerciales, se ha optado por su exclusión.

Así pues, los resultados muestran productos comerciales electrónicos con contenidos bien de información periodística (Efedata, Mynews on line, El país), bien de información especializada jurídica (Westlaw), empresarial (Axesor) o política (Fichero de altos cargos), y se han excluido diccionarios, enciclopedias y anuarios digitales (Diccionario de la RAE, Encarta).

\section{-3.1. Nivel de suscripciones de los centros de documentación}

Según los resultados mostrados en la tabla 1, los departamentos de documentación consultados tienen contratadas 16 bases de datos distintas con un total de 44 suscripciones. La media de adquisiciones por centro se sitúa por tanto entre 4 y 5 . Los centros que se aproximan más a esta media son El país y La razón con 4 contrataciones, pero sin duda la observación de los resultados muestra diferencias acentuadas entre algunos centros.

En un extremo, El mundo encabeza el ranking de manera destacada con 12 bases de datos contratadas. Le siguen también con un índice alto de adquisición de estos productos de pago El periódico de Catalunya con 7 y $A B C$ y La van-

\begin{tabular}{|c|c|c|c|c|c|}
\hline \multirow[t]{2}{*}{ Bases de datos } & \multicolumn{5}{|c|}{ Antigüedad de contratación } \\
\hline & $\begin{array}{c}\text { Menos de } 1 \\
\text { año }\end{array}$ & 1 a 3 años & 3 a 5 años & $\begin{array}{c}\text { Más de } 5 \\
\text { años }\end{array}$ & total \\
\hline$A B C$ & 1 & 1 & & & 2 \\
\hline Axesor (antes Infotel ) & & 1 & 1 & 1 & 3 \\
\hline Bloomberg & & 1 & & & 1 \\
\hline$B O E$ & & 1 & & 3 & 4 \\
\hline Dogc & & 1 & & 1 & 2 \\
\hline Efedata & & & 1 & 7 & 8 \\
\hline El País & 2 & 1 & & & 3 \\
\hline \begin{tabular}{|l} 
Factiva (antes Reuters ) \\
\end{tabular} & 1 & 1 & & 2 & 4 \\
\hline Fichero Altos Cargos & & 1 & & & 1 \\
\hline Informa & & & & 1 & 1 \\
\hline La Vanguardia & 1 & & & & 1 \\
\hline LexisNexis & & 1 & & & 1 \\
\hline \begin{tabular}{|l|} 
Mynews on line \\
\end{tabular} & & 4 & 1 & 2 & 7 \\
\hline Registro Mercantil Central & & 2 & & & 2 \\
\hline The Wall Street journal & & 1 & & & 1 \\
\hline \begin{tabular}{|l|} 
Westlaw (antes Aranzadi ) \\
\end{tabular} & 1 & & & 2 & 3 \\
\hline total & 6 & 16 & 3 & 19 & 44 \\
\hline
\end{tabular}

Tabla 2. Antigüedad de contratación de las bases de datos

guardia con 6. En el otro extremo, dos servicios de documentación consultados no disponen de ninguna suscripción, Heraldo de Aragón y La nueva España, aunque en este último caso hay que añadir que el centro manifiesta realizar consultas esporádicas y puntuales a algunas bases de datos, como Efedata y $B O E$.

\section{«El mundo encabeza el número de sus- cripciones con 12 ba- ses de datos contra- tadas y le siguen EI periódico de Catalun- ya con 7 y $A B C$ y $L a$ vanguardia con 6 »}

Una reflexión elemental que sugieren estos datos es la consideración general del bajo nivel de la utilización de fuentes electrónicas externas no gratuitas en los centros de documentación de prensa españoles, con la excepción de aquellos situados en los primeros lugares de contratación.

Precisamente el hecho de la ausencia de suscripciones a bases de datos de pago en dos centros que están situados entre los diez primeros diarios en número de lectores de España hace pensar que esta situación puede repetirse con mayor frecuencia en medios de potencial económico intermedio y constituir la realidad generalizada de los diarios más modestos.

Se puede señalar asimismo el caso singular de El país. Este medio presenta por una parte un nivel bajo de utilización de productos digitales externos (4) si se tiene en consideración que se trata del diario de información general con más lectores de España, mientras que por otro lado la comercialización reciente de su hemeroteca digital es la base de datos de un diario con más adquisiciones entre los centros de documentación de la competencia (3).

\section{-3.2. Nivel de presencia de las bases de datos}

Un segundo aspecto a considerar a partir de los datos que se presentan en la tabla 1 es el que se refiere al grado de implantación de las diferentes bases de datos. Del total de 16 productos diferentes contratados, se observa que 2 tienen una implantación muy alta: Efedata está presente en 8 de los 10 centros consultados y Mynews on line en 7. Estos dos recursos sobresalen de manera clara del resto. Todos los departamentos de documentación consultados que disponen de medios para adquirir bases de datos comerciales han adquirido Efedata y todos menos uno $M y$ news on line. 
Se puede señalar asimismo el nivel medio de implantación de algunas bases de datos altamente consideradas por los profesionales de la información, como son $B O E$ y Factiva, presentes en 4 servicios de documentación y Axesor, El Pais y Westlaw en 3 centros.

En los niveles inferiores de presencia se encuentran $A B C, \operatorname{Dog} c$ y Registro mercantil central, adquiridas cada una de ellas por 2 centros, y Bloomberg, Fichero de altos cargos, Informa, La vanguardia, LexisNexis y The Wall Street journal con una única contratación.

\section{-3. 3. Antigüedad de los productos contratados}

En la tabla 2 se presenta el grado de antigüedad de las adquisiciones de las bases de datos por los centros. El cuadro proporciona a nuestro entender como aspecto más destacable el hecho que del total de contrataciones existentes (44), cerca de la mitad (19) lo son desde un período de tiempo superior a los 5 años. El dato muestra que la utilización de las fuentes de información digitales de pago en los archivos de prensa no es una práctica documental de aparición muy reciente, sino que por el contrario se trata de una práctica generalizada en algunos centros desde hace ya varios años (si bien por nuestra parte hay que añadir que la imprecisión de las respuestas en algunos casos, no nos permite mayor exactitud en la fecha de inicio de las contrataciones más antiguas).

Entre las bases de datos externas que utilizan los centros estudiados, sobresale claramente por su veteranía Efedata: en todos ellos se utiliza desde hace más de 5 años (excepto en un diario de creación más reciente). Se puede señalar igualmente la antigüedad de algunas suscripciones de $B O E, M y$ news on line, Reuters Business Briefing y Aranzadi, antecesoras estas dos últimas respectivamente de Factiva y Westlaw. Se puede afirmar por tanto que los 5 productos electrónicos mencionados suman ya una importante tradición en la práctica documental de los servicios de documentación de los diarios españoles.

Es interesante observar también la clara evolución al alza en la alta implantación de Mynews on li$n e$ y la tendencia entre las adquisiciones más recientes de los centros de documentación a incorporar hemerotecas digitales de otros diarios, productos que sin duda han aumentado de manera visible sus prestaciones documentales.

\section{-3. 4. Comparativa de 10 años}

La ya mencionada escasez de estudios de conjunto sobre la utilización de fuentes de información en centros de documentación de prensa españoles y en concreto de bases de datos externas, impide trazar la evolución pormenorizada de la presencia de las mismas. Sin embargo, sí que podemos establecer una comparativa con diez años de diferencia observando los datos de nuestro estudio, correspondientes a agosto de 2003, y los que en su día presentaron M. Eulàlia Fuentes y Alícia Conesa, actualizados a la primavera de 1993 (Fuentes y Conesa, 1994).

Las autoras describen la situación de las prácticas documentales en diversos servicios de documentación de medios de comunicación de Catalunya, España y Europa. En lo que se refiere a prensa escrita española, analizan los centros de los diarios catalanes Avui, Diari de Barcelona, El observador, El país (edición Barcelona), El periódico y La vanguardia, así como una pequeña representación de diarios del resto de España, $A b c$ y El país.

De los diarios analizados, tan sólo 3 de ellos tenían contratadas bases de datos externas: El país utilizaba Textline, Profile e Icex, el desaparecido diario El observador poseía Efedata y Textline, y Abc tenía suscripción a Efedata. Nótese que dos centros que actualmente presentan un alto nivel de contrataciones, El periódico y La vanguardia, no poseían ninguna suscripción en 1993.

«En la última década se ha producido un incremento sustancial en la utilización de bases de datos externas comerciales por parte de los centros de documentación de prensa»

Comparando estos datos con los actuales, se constata que la utilización de bases de datos externas en los departamentos de documentación de los diarios españoles ha dado un salto considerable en 10 años, quizá con la única excepción de El país, que ya presentaba entonces un importante nivel de utilización de las mismas. Si además se toma en consideración que casi la mitad de las bases de datos utilizadas en la actualidad fueron contratadas hace más de 5 años, se puede aducir — con una cierta precaución ante la falta de datos de algunos centros- que presumiblemente una parte importante de ese "salto" en la contratación de productos digitales externos se produjo entre 1993 y 1998.

\section{Conclusiones}

En la última década se ha producido un incremento sustancial en la utilización de bases de datos externas comerciales por parte de los centros de documentación de prensa escrita en España (entendiendo por ello que nos estamos refiriendo siempre a empresas periodísticas importantes). Los niveles medios de utilización de estos productos en la actualidad se sitúan en torno a las 4 ó 5 contrataciones por de- 
partamento cuando hace diez años la mayoría de estos centros no contaba con ninguna adquisición. No obstante, la cifra indicada no impide que existan grandes diferencias entre algunos de estos centros, ya que mientras algunos servicios de documentación mantienen alto un nivel de utilización de estos productos, otros no los utilizan o lo hacen esporádicamente.

Un total de 16 bases de datos distintas constituyen en la actualidad el repertorio de productos digitales de pago que se usan documentalmente en la prensa española. De todas ellas, Efedata destaca claramente por su presencia y antigüedad hasta el punto que se puede decir de la misma que es un recurso comercial considerado prácticamente como imprescindible en aquellos centros que por sus características de disponibilidad económica pueden adquirir productos digitales de pago. Casi en los mismos términos se podría hablar de My News on line y cabe también destacar otro grupo de recursos que se pueden considerar consolidados en su alta apreciación por los documentalistas de prensa: BOE, Factiva, Axesor, Westlaw y El país. Precisamente, este último producto es un ejemplo destacado de lo que se vislumbra como una clara tendencia para el futuro inmediato: la progresiva importancia como recurso informativo de las hemerotecas digitales de los diarios, una vez han

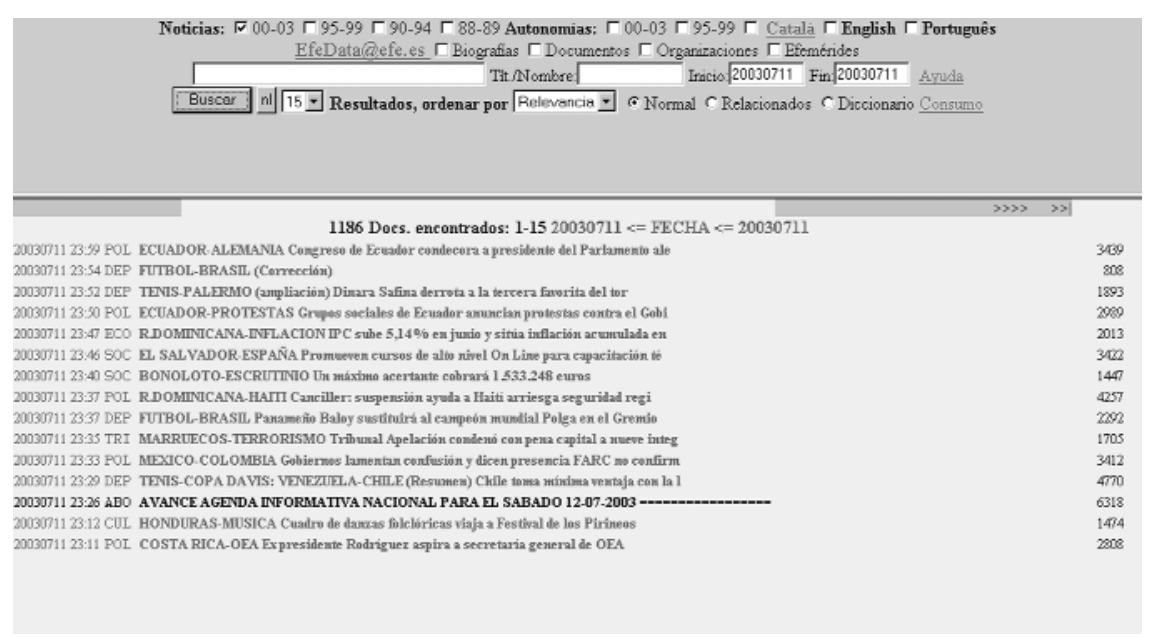

incrementado de manera importante sus prestaciones documentales.

\section{Anexo. Descripción de las bases de datos}

Como información complementaria al estudio, se muestra a continuación una breve descripción de las bases de datos comerciales que utilizan los centros de documentación de los principales diarios españoles, prestando más atención a las más contratadas.

\section{-Efedata}

Está elaborada por la agencia española de noticias $E F E$ y está considerada la mayor base de datos de noticias en español. Contiene toda la información difundida cada día por la agencia y se actualiza diariamente. Consta en realidad de varias bases de datos o secciones:

-Noticias. Está integrada por noticias de actualidad que son elaboradas por los servicios de información de la agencia EFE en todo

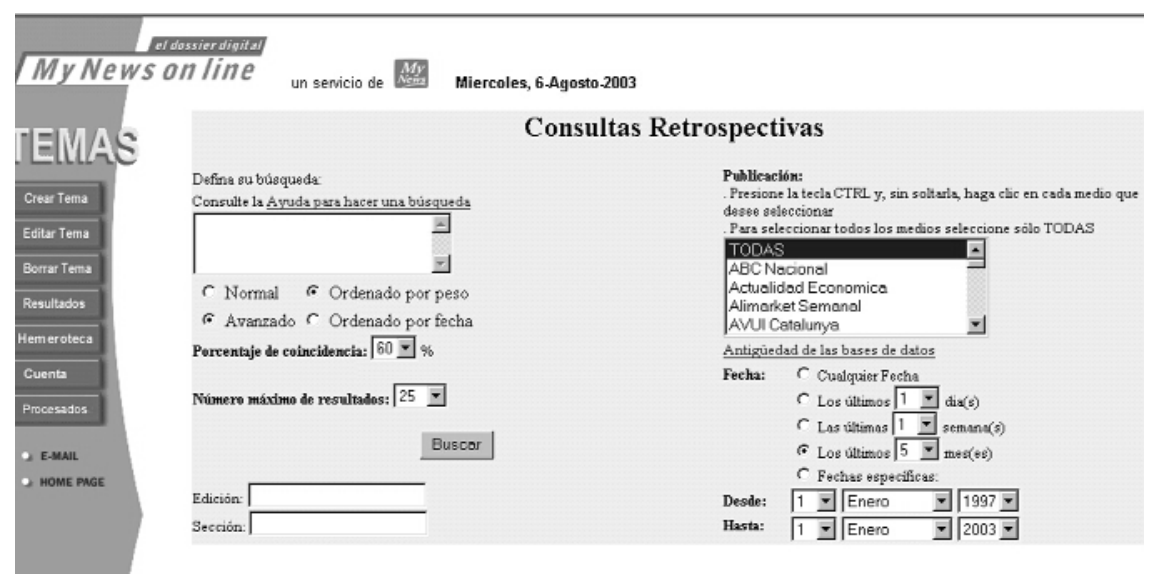

el mundo. Crece a un ritmo de 250.000 registros nuevos cada año y su cobertura temporal llega hasta 1988.

-Biografías. Contiene información sobre 12.000 personalidades españolas y extranjeras, con campos específicos de nombre, sexo, lugar y fecha de nacimiento, nacionalidad, actividad profesional, estudios o formación, organización a la que pertenecen, hechos más relevantes y la biografía completa. Indica asimismo la fecha de la última actualización de datos.

-Organizaciones. Incluye biografías de todo tipo de organizaciones que pueden ser de interés para la actividad periodística: partidos políticos, fundaciones, empresas,...

-Documentos temáticos. Tiene 3.500 registros en los que se muestran temas monográficos tratados en extensión y en muchos casos desarrollados cronológicamente.

También posee un apartado de Efemérides y una sección de Autonomías con información de interés para los medios de los diferentes ámbitos autonómicos.

http://efedata.efe.es

\section{—My News on line}

Se trata de una base de datos creada por la empresa española $M y$ News que ofrece el texto completo de varias publicaciones, en su mayoría de ámbito nacional. Aunque también dispone de servicios de 


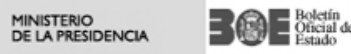

Bases de Datos del BOE

BASES DE DATOS DEL BOE ISPMI colectivos, normas UNE, ayudas ysubvenciones, etc.

-Publiboe recoge anuncios oficiales y particulares desde 1995.

-Personal incluye nombramientos, oposiciones, concursos, etc.

-Gazeta es la colección histórica digitalizada de la Gaceta de Madrid, con la mayoría de la información oficial publicada de 1914 a 1967.

Las dos primeras bases de datos, que son también las consultadas más asiduamente por los profesionales de la información, se distribuyen también en cd-rom.

http://www.boe.es

-Iberlex recoge las disposiciones de carácter general publicadas en el $B O E$ desde 1968 y la legislación europea que afecta a España publicada en el Diario Oficial de las Comunidades Europeas (Doce) desde 1986;

-Iberlex-UE contiene la legislación de las Comunidades Europeas que afecta a España publicada en el Doce;

-Indilex recoge otras disposiciones de publicación obligatoria, como planes de estudio, convenios

\section{-Factiva (antes Reuters Bu- siness Briefing)}

Es un producto conjunto de la agencia de noticias Reuters y de la empresa Dow Jones, editora de The World Street journal. Funciona desde 1999 como resultado de la fusión de la conocida base de datos Reuters Business Briefing con productos informativos de Dow Jones.

Proporciona información de índole diversa, pero destaca especialmente en la información económica y de noticias. Las fuentes Expansión, La vanguardia y Mar$c a$, entre otros). A partir de ahí se han ido ampliando y ésta es una de las razones de su éxito. El año 2003 My News ha incorporado a su base de datos Diari de Balears, Diario de noticias, Última Hora y Noticias de la agencia Efe.

http://www.mynewsonline.com

\section{-Boletín Oficial del Estado (BOE)}

La página web del organismo español que se encarga de la publicación de leyes, disposiciones y actas de inserción obligatoria ofrece acceso gratuito al boletín del día y a la consulta de los sumarios de los boletines desde 1995, mientras que las consultas retrospectivas de

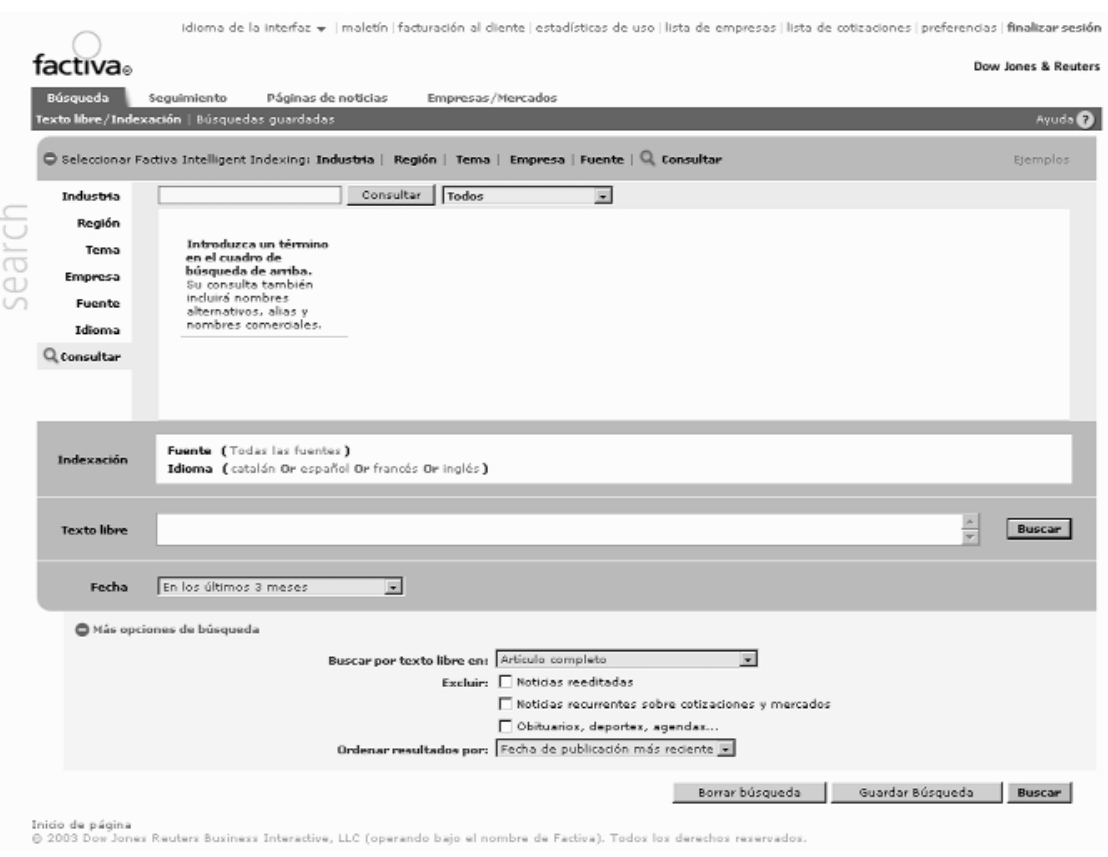


que utiliza son por una parte la propia agencia de noticias Reuters y por otra parte una ingente cantidad de publicaciones de todo el mundo: unas 8.000 , de las cuales 122 son en español.

Se puede consultar en diferentes idiomas, entre ellos el español. Tiene unas prestaciones de búsqueda muy altas, tanto por la magnitud de su fondo documental como por las facilidades que ofrece de personalización de búsquedas (preselección de fuentes de información, diferentes interfaces, etc.)

Los documentalistas de los medios de prensa escrita la utilizan también como complemento a las bases de datos nacionales para las consultas de ámbito español, pero sin duda su mayor potencial se encuentra en las búsquedas informativas de temática internacional.

http://global.factiva.com

\section{-Axesor (antes Infotel)}

Es una base de datos creada por Axesor, empresa fundada en 1996 con el nombre de Infotel y con sedes en España y Argentina que se ha especializado en ofrecer servicios de información empresarial a través de internet. Divide su contenido en 5 grandes áreas: Información de empresas, Marcas y dominios, Gestoría virtual, Marketing, Boletines e Instrumentos Jurídicos.

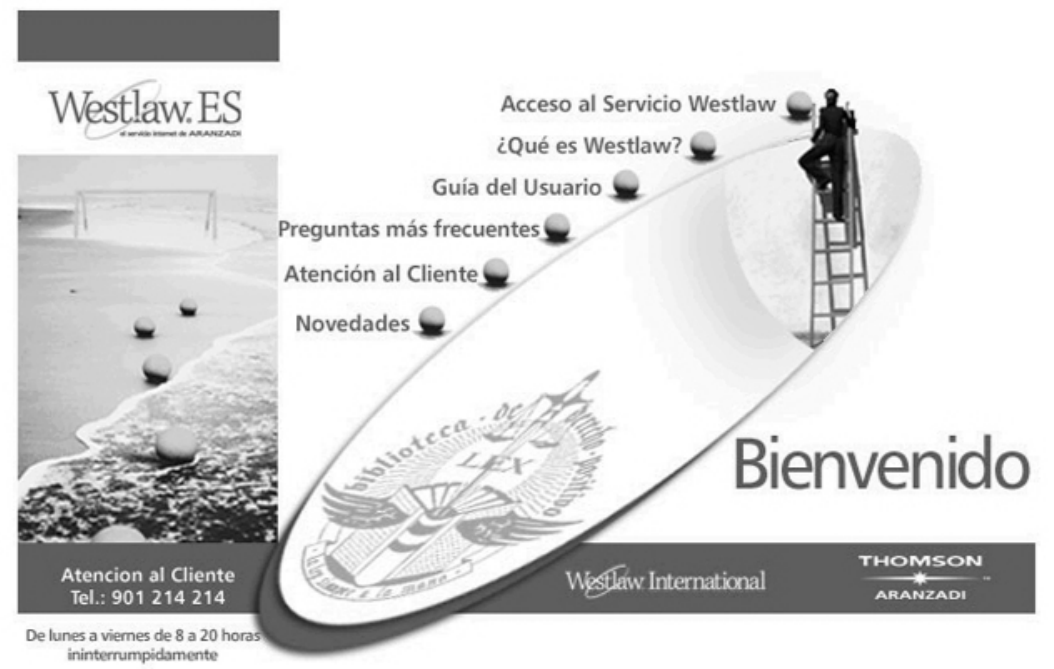

La primera área es la de mayor interés para un centro de documentación de prensa por su contenido de informes comerciales, datos económicos, financieros y mercantiles, demandas judiciales y noticias. Su utilización por los centros está relacionada tanto con la publicación de noticias sobre empresas como con el desarrollo del periodismo de investigación: permite indagar, por ejemplo, a qué empresas está vinculada una persona o si una compañía ha tenido alguna demanda judicial.

http://www.axesores

\section{-El país}

El web del diario ofrece por suscripción desde noviembre de 2002 una hemeroteca digital que

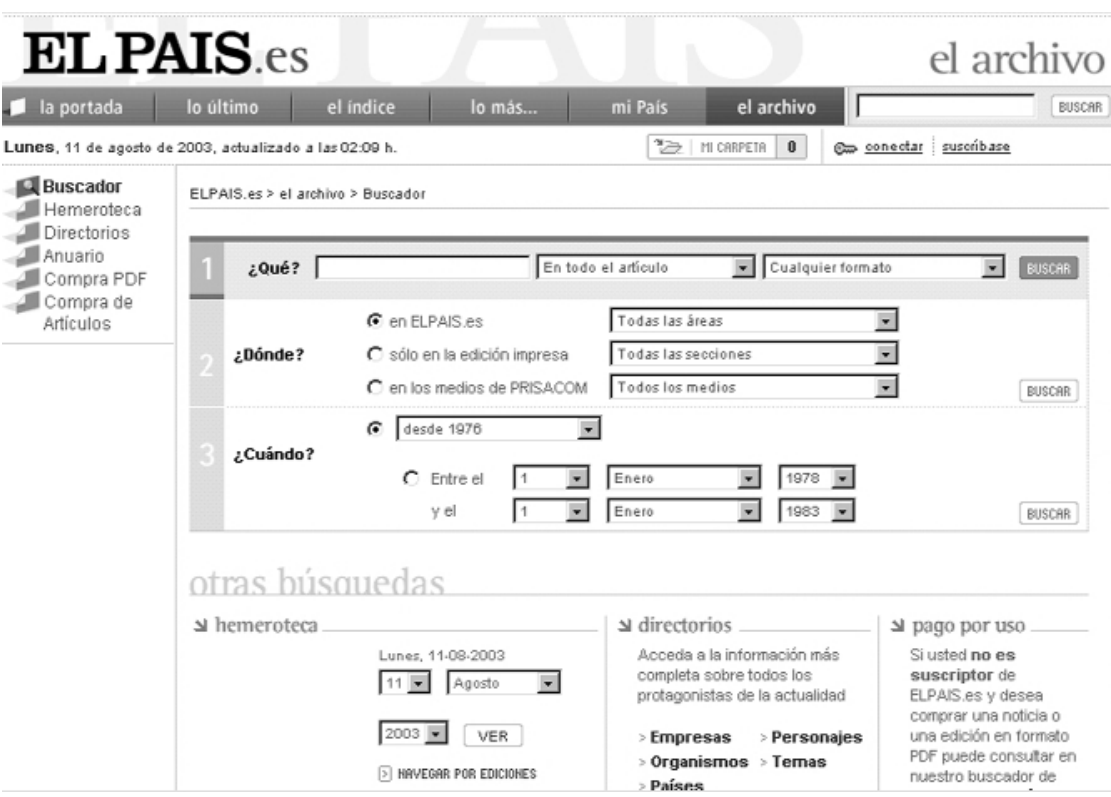

ha tenido una importante repercusión entre los medios. Desde el punto de vista documental, es una interesante fuente de información externa para los centros de documentación de otros diarios.

Su principal interés es el acceso al contenido íntegro de la edición impresa del diario así como a los cuadernillos de las ediciones autonómicas y la mayoría de sus suplementos desde su fundación el 4 de mayo de 1976. También permite acceder a otros medios del grupo Prisa como 5 Días o el diario As.

Se pueden acotar las búsquedas por formatos, por intervalos temporales, por áreas informativas del web, por secciones de la edición impresa o por medios del grupo Prisa.

Al igual que sucede con otros diarios, El país ha comercializado hasta ahora también sus fondos en soporte cd-rom, y falta saber si el éxito de la versión en internet puede tener consecuencias en la continuidad del soporte óptico.

http://www.elpais.es

\section{—Westlaw (antes Aranzadi)}

Es una base de datos de legislación y jurisprudencia desarrollada conjuntamente por la editorial 


\section{SISTIEMIA SAIBIINI}

\section{Soluciones integrales para la Automatización de Bibliotecas y Centros de Documentación}

Desarrollado integramente en castellano con más de 15 años de experiencia en España y

Latinoamérica, atiende las necesidades de todo tipo y tamaño de bibliotecas y centros de información

MÓDULOS:

- Adquisiciones

Trămite de pexfidos

Gesticon de gastos y proveedores

- Catalogación

Catalogacion de todos los materlales biloliográticos Integración de información bibliográfica

- Terminologia

Ficheros de auloridades

Tesauro multiingüe

Lenguaje de Comandos

- Consultas

Acceso público en linea (CAPEL)

Acceso a través de WWW

Protocolo 739.50

- Circulación

Gestión de préstamos

Fichero de usuarios

- Estadísticas

De proceso y circulación

Otros servicios:

Instalación y soporte técnico del sistema SABINI

Asesoria en Automatización de Bibliotecas y Centros de Documentación

Procesamiento de material bibliográfico

Instalación de catálogos en Internet de Bibliotecas
Cr Amar de Dica, $\mathrm{n}^{*} 1$ Tolf.: +34 91429055 $\mathrm{Fax}+34914292010$ 20014 MMRID F-mal: sabunfistabion

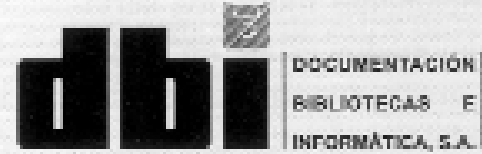

Av. Diez Cinseco Zse of. 602 Lims 18 Twhere: (511) 446-0815 en-mail: anbinipterra.compe 
española Aranzadi, especializada en información de este ámbito, y el grupo multinacional The Thomson Corporation Proporciona el conjunto de fuentes más completo de ámbito nacional para las necesidades de documentación periodística de índole legislativo y jurídico.

Entre otras, contiene las siguientes secciones: Legislación estatal (texto completo de las disposiciones publicadas en el $B O E$ desde 1978 e información referencial hasta 1930); Legislación autonómica (todas las disposiciones publicadas por las diferentes comunidades autonómicas); Legislación europea (selección desde 1952); Legislación consolidada básica (selección de la legislación más utilizada y relevante desde 1829); Selección Aranzadi (las resoluciones más relevantes dictadas por los tribunales); Bibliografía (25.000 referencias bibliográficas de las que 3.000 ofrece el texto completo).

http://www.westlaw.es

$$
-\boldsymbol{A B C}
$$

La página web del diario proporciona el acceso gratuito a la información publicada en los últimos días y al servicio de búsquedas en su hemeroteca, mientras que el contenido de los artículos es de pago. El archivo digital en internet contiene noticias desde 1996. Por otra parte, $A B C$ ofrece también la colección del diario en soporte cdrom.

http://www.abc.es

\section{-Diari Oficial de la Genera- litat de Catalunya (Dogc)}

La base de datos del órgano oficial de publicación de la legislación catalana tiene lógicamente un interés mayor para los centros de este ámbito autonómico (las dos suscripciones corresponden a los dos diarios catalanes consultados). El Dogc proporciona acceso a través de internet a los documentos publicados desde su primer núme- ro (5 de diciembre de 1977) y es asimismo consultable en cd-rom. http://www.gencat.es/diari/

\section{—Registro Mercantil Central}

Es una institución oficial española de publicidad que permite en su base de datos el acceso a la información mercantil que suministran los Registros provinciales desde 1990. Su utilización en prensa está relacionada con la búsqueda de información sobre empresas y el periodismo de investigación.

http://www.rmc.es

\section{-Bloomberg}

Es un producto altamente especializado de información financiera. Algunos de sus puntos fuertes son la información instantánea de los mercados las 24 horas del día y las herramientas profesionales para el análisis de la bolsa. Contiene asimismo textos de unas 850 fuentes de información económica.

http://www.bloomberg.com

\section{-Fichero de Altos Cargos (FAC)}

Es un directorio de altos cargos de la administración central y de las administraciones autonómicas elaborado por la empresa española Ficesa. Se trata de un producto de consulta muy utilizado en los diarios en su formato tradicional de fichas de papel actualizadas mensualmente. Desde finales de 1999 se distribuye también en cdrom, formato en el que sólo uno de los centros consultados lo ha adquirido.

http://www.fac-ficesa.com

\section{- Informa}

$\mathrm{Al}$ igual que Axesor, Informa es un producto dedicado a suministrar información económica y financiera de empresas por internet. Tiene censados unos 4,5 millones de agentes económicos (compañías). Ofrece informes comerciales y servicios de vigilancia a empresas. Su utilización documental en pren- sa se sitúa en la línea de lo dicho para Axesor.

http://www.informasa.net

\section{- La vanguardia}

La edición digital del diario $\mathrm{La}$ vanguardia, del Grupo Godó, permite la búsqueda retrospectiva de noticias desde 1999. Además, recientemente ha añadido el importante fondo histórico del diario que se remonta a 1881 , aunque por el momento solamente es consultable por fechas de edición en formato pdf.

http://www.lavanguardia.es

\section{—LexisNexis}

Es un producto informativo de altas prestaciones del grupo empresarial del mismo nombre perteneciente a la empresa anglo-holandesa Reed Elsevier. Proporciona información económica, empresarial, fiscal, legislativa y de actualidad internacional con un total de unos 3.000 millones de documentos procedentes de más de 31.000 fuentes. Sorprende que su elevado potencial solamente sea utilizado por un centro de los consultados. http://www.lexisnexis.com

\section{-The Wall Street journal}

La edición en línea del conocido diario norteamericano perteneciente al grupo Dow Jones es un interesante recurso de información económica y financiera internacional. Además de las noticias de actualidad económica, ofrece información de mercados financieros e informes comerciales de empresas. http://www.wsj.com

\section{Nota}

Los autores quieren agradecer las facilidades y la colaboración prestadas por los centros de documentación consultados.

\section{Bibliografía}

Aimc-EGM. Ranking de medios impresos. En: EGM octubre de 2002 a mayo de 2003 Resumen general Consultado en: 25-07-03.

http://download.aimc.es/aimc/02egm/resumene gm6.pdf 
Carlino, Luis. "Reuters business briefing (RBB), la base de datos de Reuters". En: Cuadernos de documentación multimedia, 19971998. Consultado en: 28-07-03.

http://www.ucm.es/info/multidoc/multidoc/revist a/cuad6-7/rbb.htm

Cebrián Enrique, Bernardino. Fuentes de consulta para la documentación informativa. Madrid: Cees Ediciones, 1997.

Cebrián Enrique, Bernardino. "Las obras de consulta”. En: Galdón, Gabriel (coord.): Teoría y práctica de la documentación informativa. Barcelona: Ariel, 2002, pp. 121-136.

Codina, Lluís. Sistemes d'informació documental. Barcelona: Pòrtic, 1993.

Chacón Gutiérrez, Inmaculada. “Tipología y fiabilidad de las fuentes documentales". En: García Gutiérrez, Antonio (ed.): Introducción a la documentación informativa y periodística. Sevilla: Mad, 1999, pp. 63-86.

Chacón Gutiérrez, Inmaculada; García Jiménez, Antonio. "Documentación para el periodismo especializado". En: Revista general de información y documentación, 2001, vol. 11 , n. 2, pp. 33-60

Delgado Soler, Cinta. "Documentación de prensa". En: García Gutiérrez, Antonio (ed.): Introducción a la documentación informativa y periodística. Sevilla: Mad, 1999, pp. 450-469.

El País. "El nuevo El País digital da plena soberanía al lector para consultar la información". En: El país, 21 de noviembre de 2002, Ciberp@is, pp. 8-9.

Fuentes i Pujol, M. Eulàlia. "Els serveis de documentació dels diaris de Barcelona: deu anys després". En: Jornades catalanes de documentació. Biblioteques, centres de documentació o serveis d'informació. Barcelona 1992 Barcelona: 1992, pp. 285-305.
Fuentes i Pujol, M. Eulàlia. "Evolució de la documentació periodística a Espanya durant els darrers cinc anys i algunes experiències europees". En: Seminari La documentació als mitjans d'informació. $2^{\circ}$. 1994. Valencia: Generalitat Valenciana; Unió de Periodistes, 1994, pp. 22-27.

Fuentes i Pujol, M. Eulàlia. "Características generales de la documentación periodística y características específicas de los medios de comunicación escritos" En: Fuentes i Pujol, $\mathbf{M}^{\mathrm{a}}$ Eulalia (ed.): Manual de documentación periodística. Madrid: Síntesis, 1995, pp. 135-145.

Fuentes i Pujol, M. Eulàlia; Conesa, Alícia. La documentació periodística. Catalunya, Espanya $i$ altres experiències europees. Barcelona: Centre d'Investigació de la Comunicació, 1994

Fuentes i Pujol, M. Eulàlia; González Quesada, Alfons; Jiménez López, Àngels. "Documentación e información electrónica”. En: Moreiro, José Antonio (coord.): Manual de documentación informativa Madrid: Cátedra, 2000, pp. $345-422$.

Fuentes i Pujol, M. Eulàlia; González Quesada, Alfons; Jiménez López, Àngels. "Servicios y sistemas de acceso a la información periodística en internet". En: Fuentes i Pujol, M Eulàlia (dir.): Anuari de Biblioteconomia, Documentació i Informació Bibliodoc 2000. Barcelona: COBDC, 2001, pp. 85-102.

Galdón López, Gabriel. "Fuentes necesarias y modos de utilizarlas". En: Galdón López, Gabriel. El servicio de documentación de prensa: Funciones y métodos. Barcelona: Mitre, 1986.

Giménez Toledo, Elea. "Las bases de datos para los profesionales de la comunicación". En: Galdón, Gabriel (coord.): Teoría y práctica de la documentación informativa. Barcelona: Ariel, 2002, pp. 175-196.

Guillem Bach, Carlos. "Prensa a la carta: situación actual y posible evolución”. En: $\mathrm{Cu}$ - dernos de documentación multimedia, 19971998. Consultado en: 28-07-03.

http://www.ucm.es/info/multidoc/multidoc/revist a/cuad6-7/rbb.htm

Hernández Pérez, Antonio; Caridad Sebastián, Mercedes. "Documentación escrita en los centros de documentación de los medios de comunicación". En: Moreiro, José Antonio (coord.): Manual de documentación informativa. Madrid: Cátedra, 2000, pp. 37-82.

Marcos, Mari Carmen; Baiget, Tomàs. "Integración y personalización de contenidos, la clave del éxito de Factiva". En: El profesional de la información, 2003, enero-febrero, v. 12, n. 1, pp. 26-30.

Pareja Pérez, Víctor Manuel (coord.). Guía de internet para periodistas. Madrid: Centro de Información y Documentación Cindoc (CSIC), 2002, 196 p.

Pons Serra, Amadeu. "Fuentes de información para la actividad periodística". En: Fuentes i Pujol, M. Eulàlia (ed.): Manual de documentación periodística. Madrid: Síntesis, 1995. pp. 53-76.

Quesada Pérez, Montserrat. "Necesidades documentales de la profesión de periodista". En: Fuentes i Pujol, M. Eulàlia (ed.): Manual de documentación periodística. Madrid: Síntesis, 1995, pp. 45-52.

Rubio Lacoba, María. "Otras fuentes en internet”. En: Galdón, Gabriel (coord.): Teoría y práctica de la documentación informativa. Barcelona: Ariel, 2002, pp. 207-219.

Javier Guallar Delgado y Anna Cornet Casals El periódico de Catalunya. Departamento de documentación. jguallard@uoc.edu jguallar@elperiodico.com acornet@elperiodico.com

Taylor \& Francis The Netherlands, editora de esta revista, tiene encargada la distribución de sus publicaciones a la siguiente empresa:

Extenza-Turpin. Blackhorse Road, Letchworth, SG6 IHN, Herts, Reino Unido.

\author{
Tel.: +44-I46267 2555; fax: I462480947 \\ subscriptions@extenza-turpin.com
}

Rogamos a nuestros suscriptores que para solventar cualquier asunto administrativo se dirijan siempre directamente a Extenza-Turpin. Recordamos que continúan en funcionamiento los números de teléfono de atención al suscriptor en Barcelona:

Tel.: +34-932 08I 970; fax: 932 08I 97| 